\title{
Diagnosis of renal transplant rejection: Banff classification and beyond
}

\author{
Hyeon Joo Jeong ${ }^{(10)}$ \\ Department of Pathology, Yonsei University College of Medicine, Seoul, Republic of Korea
}

\begin{abstract}
Diagnosis of renal transplant rejection is dependent on interpretation of renal allograft biopsies. The Banff Classification of Allograft Pathology, which was developed as a standardized working classification system in 1991 , has contributed to the standardization of definitions for histologic injuries resulting from renal allograft rejections and provided a universal grading system for assessing these injuries. It has also helped to provide insight into the underlying pathogenic mechanisms that contribute to transplant rejection. In addition to histological and immunologic parameters, molecular tools are now being used to facilitate the diagnosis of rejection. In this review, I will discuss morphologic features of renal transplant rejections as well as major revisions and pitfalls of the Banff classification system, and provide future perspectives.
\end{abstract}

Keywords: Banff classification, Gene transcripts, Morphology, Renal transplantation, Rejection

\section{Overview of renal transplant rejection}

Renal transplantation is an effective treatment option for patients with end-stage renal disease. Since the first successful renal transplant, which was carried out between identical twins in 1954 in the United States [1], this procedure has been widely performed in many Western and Eastern countries. In Korea, the first successful renal transplant occurred in 1969, and in the past six years, more than 15,000 renal transplants have been performed [2].

The concept of rejection was recognized in the early

Received January 8, 2020; Revised February 10, 2020;

Accepted February 19, 2020

Edited by Beom Jin Lim, Yonsei University, Seoul, Republic of Korea Correspondence: Hyeon Joo Jeong

Department of Pathology, Yonsei University College of Medicine, 50-1 Yonsei-ro, Seodaemun-gu, Seoul 03722, Republic of Korea. E-mail:jeong10@yuhs.ac

Copyright () 2020 by The Korean Society of Nephrology

(a) This is an open-access article distributed under the terms of the Creative Commons Attribution Non-Commercial License (http://creativecommons. org/licenses/by-nc-nd/4.0/), which permits unrestricted non-commercial use, distribution, and reproduction in any medium, provided the original work is properly cited. 1900s by Alexis Carrell, who coined the term 'biologic incompatibility' [1]. Rejection is an immunologic reaction to donor antigens that are recognized by a recipient's immune system. This continues to present a major obstacle to long-term allograft survival [3], despite progressive improvements in surgical techniques, advances in immunology, and the introduction of new immunosuppressive drugs. Renal allograft biopsy is a direct and confirmative tool for the diagnosis of rejection that can be used to assess both the type and degree of rejection.

Pathologic changes associated with acute and chronic renal allograft rejections have been reported since the late 1960s [4-7]. However, no standardized classification system was proposed until 1991, when a group of 28 renal pathologists, nephrologists, and transplant surgeons gathered in Banff, Canada, and outlined international standards for the definition and grading of transplant rejection [8]. After the first 'Banff Working Classification of Kidney Transplant Pathology' report, successive biannual meetings have been held, with the recent 2019 meeting taking place in Pittsburgh [9-18], and the results have been published as meeting reports. In this review, I will discuss morphologic features of renal transplant 
rejections, up-to-date revisions and pitfalls of the Banff Classification of Allograft Pathology [8-18], and future perspectives.

\section{Pathology of renal allograft rejection}

Rejection pathology can be observed in all four components of the kidney-the glomeruli, tubules, interstitium, and vessels-either individually or in combination. In most rejection cases, renal allograft biopsy shows morphologic injuries resulting from predominantly cellular or antibody-mediated mechanisms. Depending on the time post-transplantation and rejection activity, this condition can be classified as either acute/active or chronic. However, acute and chronic changes can exist within the same biopsy [19]. Importantly, although acute and chronic are clinical terms suggesting the time of onset of a disease, here, this classification reflects activity or inactivity, rather than time of biopsy. Thus, active lesions may appear late, and chronic lesions may develop early during the post-transplant period.

Interpretation of allograft histology should consider recipient as well as donor factors. For example, older donors may have significant glomerulosclerosis and tubulointerstitial fibrosis, and kidneys from brain-dead donors may have ischemic changes. Rarely, donor-transmitted disease may be observed in time-zero or early biopsies. In this case, an implantation or zero-hour biopsy may be useful as a reference. In late-transplant biopsies, the possibility of recurrent or de novo development of glomerular, metabolic, or systemic diseases should be considered. Drug toxicity and infections may also develop at any time post-transplant. If a graft biopsy is obtained late, graft histology is likely to show mixed features that are attributable to more than one cause.

Renal allograft biopsy samples can be analyzed by light, immunofluorescence, and electron microscopy. Among these, the features that are assessed by light microscopy are essential and the most important. Immunofluorescence microscopy or immunohistochemistry is needed to detect footprints of antibody binding and immune complexes, whereas electron microscopy is used for the detection of chronic antibody-mediated rejection (ABMR). Rejection pathology can be described according to activity or the histologic component involved, as described below.

\section{Rejection pathology according to activity}

\section{Acute (active) rejection}

Acute (active) rejection is characterized by tubulitis, interstitial inflammation, glomerulitis, peritubular capillaritis, and arteritis.

\section{Chronic rejection}

Chronic rejection is characterized by tubular atrophy, interstitial fibrosis, transplant glomerulopathy, multilayering of peritubular capillary (PTC) basement membranes, and transplant arteriopathy.

\section{Rejection pathology according to histologic component}

\section{Glomerulus}

\section{Glomerulitis}

Glomerulitis is characterized by endothelial enlargement and inflammatory cell infiltration, often resulting in capillary luminal narrowing and destruction (Fig. 1). The infiltrating inflammatory cells may be T cells, monocytes, or neutrophils. This can be observed in the context of ABMR, and it is believed to be caused by endothelial injury that is mainly directed to human leukocyte antigen (HLA).

Inflammatory cells may also be present in non-ABMR conditions, such as acute $\mathrm{T}$ cell-mediated rejection

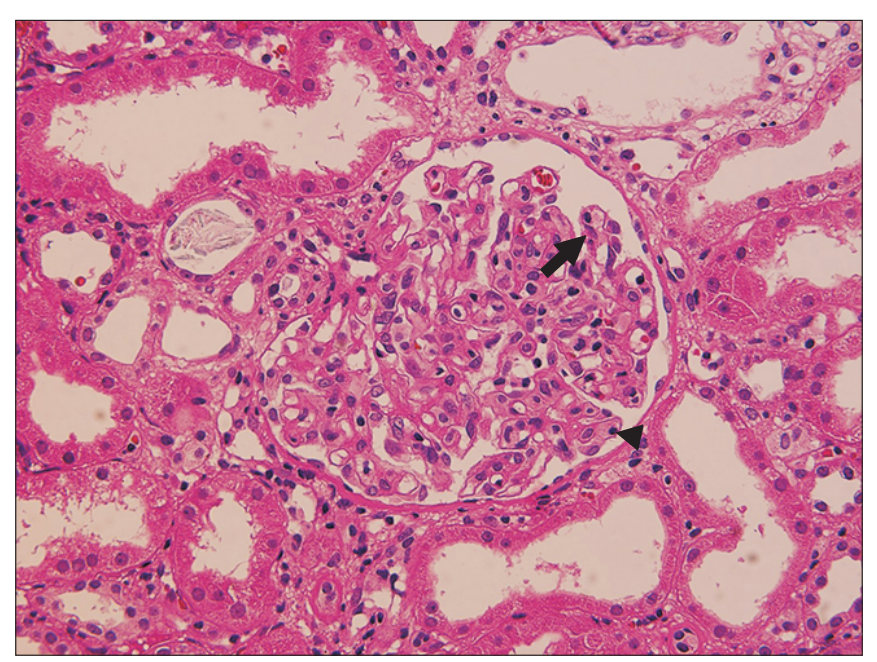

Figure 1. A glomerulus shows hypercellularity, with endothelial swelling (arrow) and inflammatory cell infiltration (arrowhead), as detected by hematoxylin and eosin $(\mathrm{H} \& \mathrm{E}, \times 200)$ staining. 


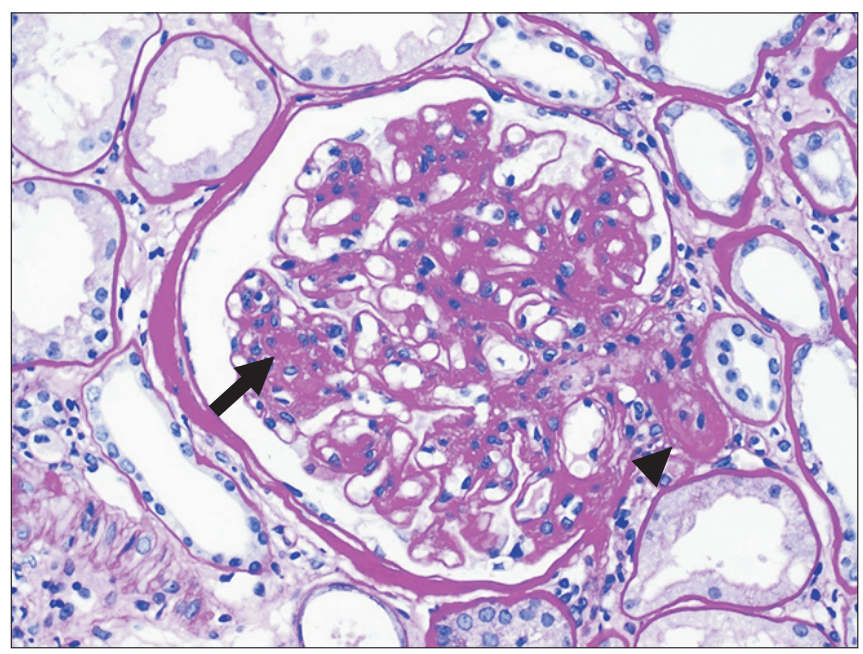

Figure 2. Periodic acid-Schiff stain shows mesangial expansion (arrow), with increased mesangial cells and matrix. Afferent arteriolar hyalinosis (arrowhead) is also present $(\times 200)$.

(TCMR) and glomerulonephritis. For example, glomerular hypercellularity, which is referred to as 'endocapillary hypercellularity', is sometimes observed in immunoglobulin (Ig)A nephropathy that develops after transplantation [20]. This can therefore produce a diagnostic dilemma, particularly when the presence of concurrent ABMR is suspected.

\section{Mesangiolysis}

Mesangiolysis results from dissolution of the mesangial matrix and manifests as a pale area after periodic acidSchiff (PAS) staining. It may be present in the context of ABMR, but may also occur with non-rejection conditions that are associated with endothelial or mesangial cell injury. The most common condition in which this occurs is thrombotic microangiopathy (TMA), but mesangiolysis may also be present in other glomerular diseases.

\section{Mesangial matrix increase}

An increased mesangial matrix is defined as a matrix that exceeds the width of two mesangial cells in two adjacent glomerular lobules (Fig. 2). The mesangial matrix may be increased in association with chronic rejection; however, this feature is entirely nonspecific. In practice, increased mesangial matrix, along with mesangial hypercellularity, is frequently associated with IgA nephropathy or diabetic nephropathy post-transplant.

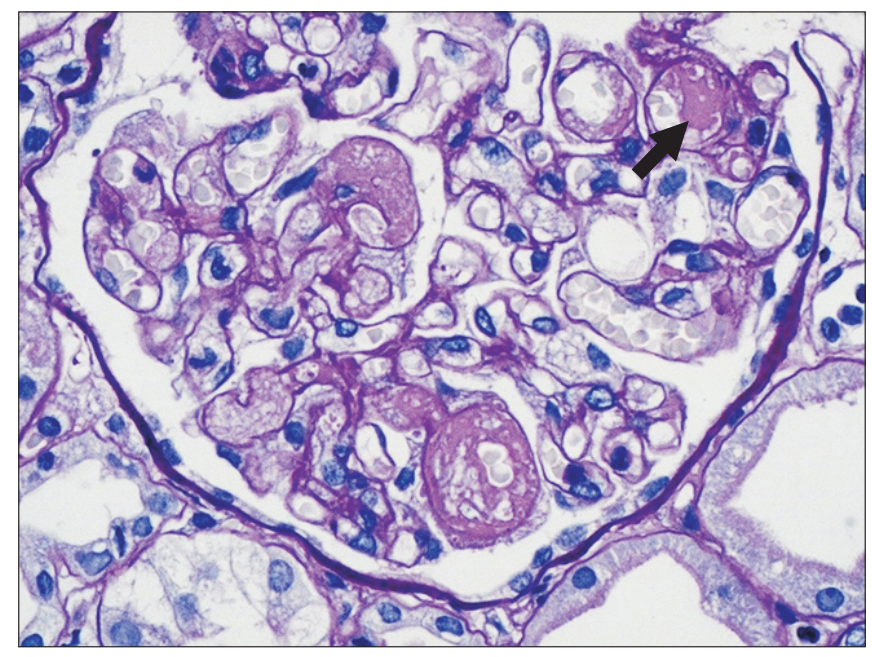

Figure 3. Several glomerular capillary lumens showing microaneurysmal dilatation; these lumens are filled with pinkish fibrinous material (arrow) as detected by periodic acid-Schiff staining $(\times 400)$.

TMA

TMA is characterized by microthrombi, glomerular subendothelial electron-lucent widening, deposition of fluffy material, and the formation of a new subendothelial basement membrane (Fig. 3). This can be observed in the context of active ABMR related to endothelial injury. However, TMA may be present in other non-rejection conditions, such as recurrent atypical hemolytic uremic syndrome or drug-related conditions. In particular, calcineurin inhibitors, such as cyclosporine A and tacrolimus, induce dose-dependent endothelial dysfunction [21,22], and sirolimus, administered either alone or in combination with cyclosporine, can cause TMA [23]. Therefore, differential diagnosis may not be possible without both clinical history and laboratory data.

\section{Transplant glomerulopathy}

Transplant glomerulopathy is characterized by doubling or even multilayering of the glomerular basement membrane (GBM) (Fig. 4). GBM doubling is best detected with PAS or methenamine silver methods, but is only demonstrable by electron microscopy in the early stages of development. Transplant glomerulopathy may be seen in chronic ABMR that is caused by repeated endothelial injury and repair. Similar features can be present in other conditions, such as TMA and membranoproliferative glomerulonephritis associated with hepatitis $C$ viral infection. However, glomerular immune complex deposition is a feature of membranoproliferative glomerulonephri- 

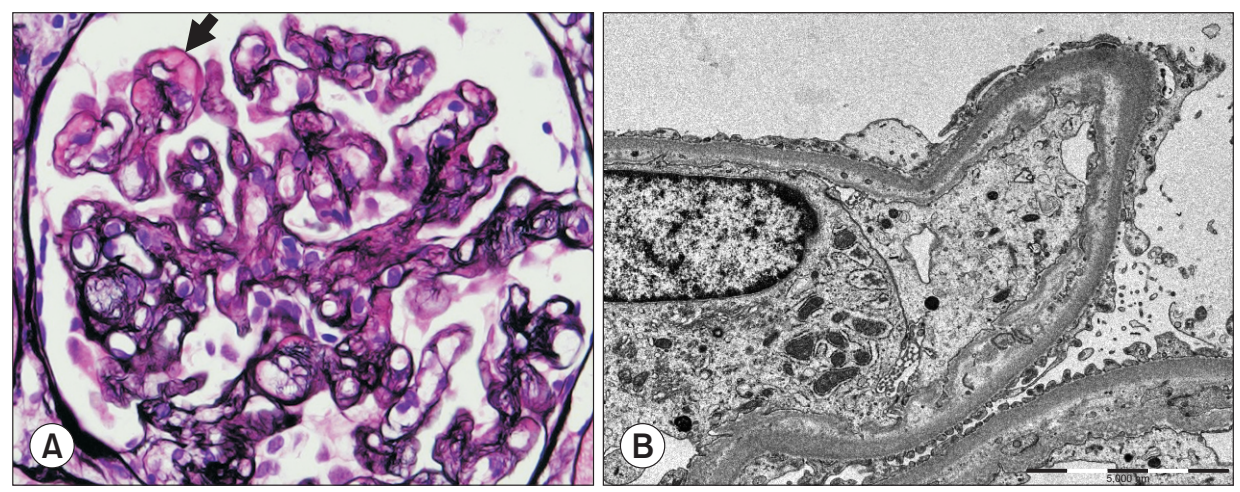

Figure 4. (A) Thickening and doubling of the glomerular basement membrane (arrow) is detected in transplant glomerulopathy (methenamine silver staining, $\times 200)$. (B) Subendothelial electronlucent widening and new basement membrane formation can be seen.

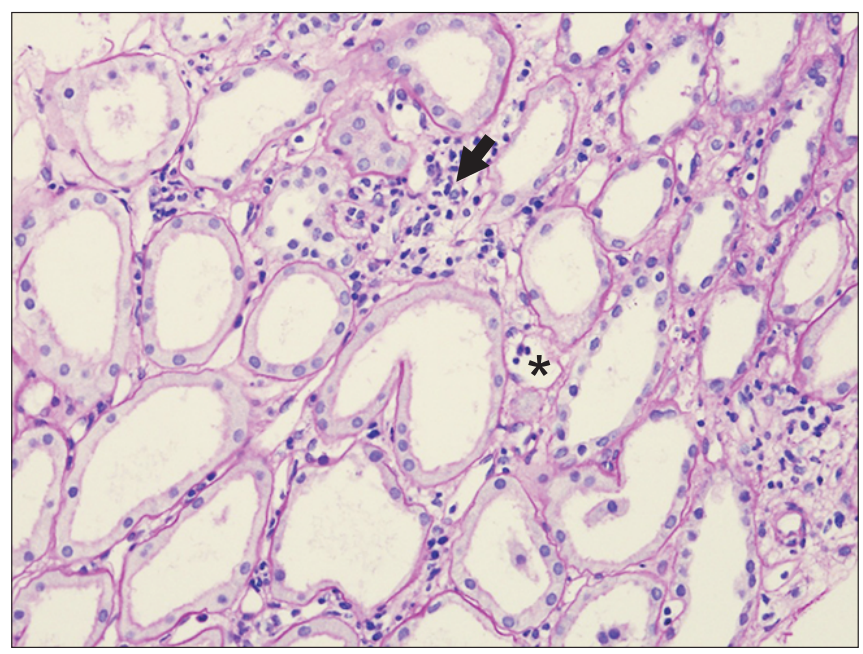

Figure 5. Tubules show flattening of the cytoplasm and luminal dilatation, associated with interstitial edema, mild interstitial inflammation (arrow), and peritubular capillaritis (asterisk) (periodic acidSchiff staining, $\times 200)$.

tis, whereas IgM and C3 deposits may be present in transplant glomerulopathy.

\section{Tubules}

Tubular degeneration/necrosis

Acute tubular injury may be a sign of active ABMR if it is present in the absence of other known causes. Proximal tubular epithelial cells are most commonly affected, and flattening of the cytoplasm, loss of brush borders, and luminal dilatation can be observed (Fig. 5). Overt necrosis is not common, and intratubular microcalcification may be present.

Overt tubular necrosis is also often associated with polyomavirus infection. In this case, necrotic tubular cells may have intranuclear viral inclusions. In addition,

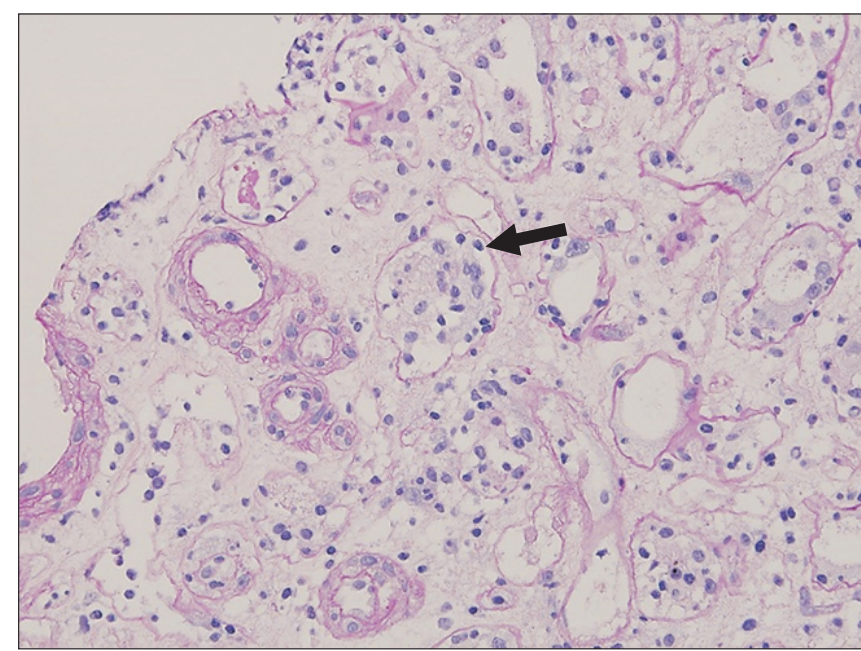

Figure 6. Mononuclear cells (arrow) are present between tubular epithelial cells in acute T cell-mediated rejection (periodic acid-Schiff staining, $\times 200$ ).

acute tubular injury may be a sign of ischemia or drug toxicity. Therefore, observation of other clinical or immunologic features supportive of ABMR is required to confirm diagnosis.

\section{Tubulitis}

Tubulitis is a feature of acute and chronic active TCMR. It is characterized by the presence of inflammatory cells between tubular epithelial cells (Fig. 6). In acute TCMR, tubulitis is present in non-severely atrophic tubules. In chronic active TCMR, tubulitis involves both atrophic and non-atrophic tubules. The inflammatory cells present in tubulitis are most commonly lymphocytes and monocytes, but plasma cells may also be present.

Tubulitis can further result from infections and druginduced interstitial nephritis, and it occasionally occurs in association with glomerulonephritis. Neutrophils may 


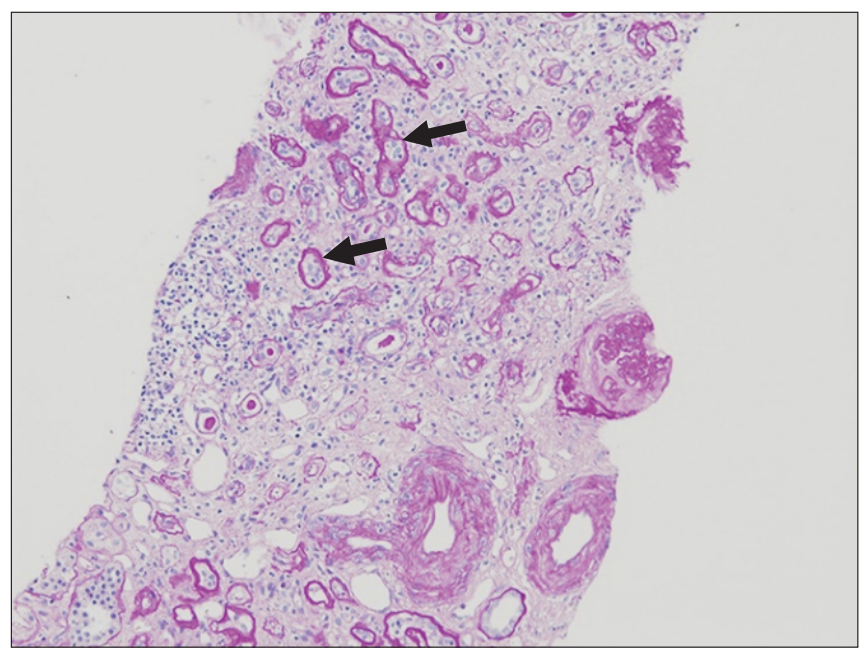

Figure 7. Periodic acid-Schiff staining shows reduced tubular sizes and thickening of tubular basement membranes (arrows), compatible with tubular atrophy $(\times 40)$.

be dominant in bacterial infection, often with intraluminal neutrophilic abscess, and plasma cells are sometimes present in infiltrate from polyomavirus-associated interstitial nephritis. However, distinction between druginduced interstitial nephritis and TCMR is not always possible.

\section{Tubular atrophy}

Tubular atrophy is a non-specific feature of chronic rejections and non-rejections. This is characterized by a $>50 \%$ narrowing of the tubular diameter or thickening of the basement membrane (Fig. 7). In some cases, this condition resembles endocrine glands without thickening of basement membrane. Severity of tubular atrophy is divided into three tiers (mild, moderate, and severe) based on the percentage of atrophic tubules $(<25 \%, 25 \%$ to $50 \%$, and $>50 \%$ ), and atrophic tubules are frequently replaced by interstitial fibrosis.

\section{Interstitium}

\section{Interstitial edema}

Interstitial edema is characterized by the presence of widened and lightly stained interstitium due to the accumulation of tissue fluid (Fig. 8). In acute TCMR, inflammatory cells are present in the edematous stroma.

Interstitial inflammation

Interstitial inflammation is a feature of acute and

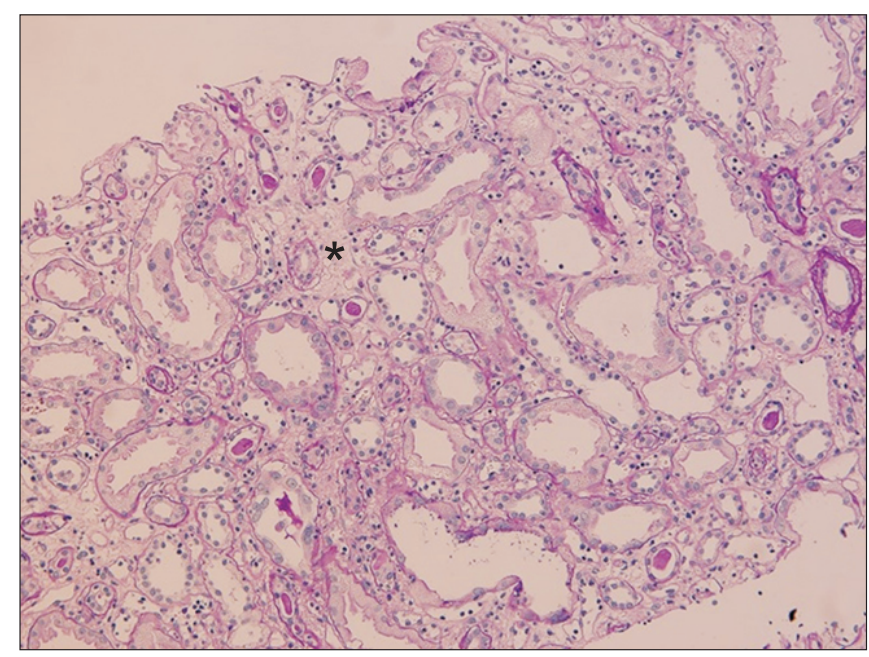

Figure 8. Acute tubular injury, interstitial edema (asterisk), minimal interstitial inflammation and peritubular capillaritis are observed (periodic acid-Schiff staining, $\times 100$ ).

chronic active TCMR. In acute TCMR, inflammatory cell infiltration is present in the edematous stroma, associated with tubulitis of non-severely atrophic tubules (Fig. 8). In chronic active TCMR, inflammatory cells are found in both the edematous and fibrotic stroma, and this is accompanied by tubulitis of atrophic and non-atrophic tubules. The infiltrating cells are most often lymphocytes and monocytes, but plasma cells, neutrophils, or eosinophils can also be present. Immunohistochemistry has shown that $\mathrm{T}$ cells tend to infiltrate diffusely or in scattered patterns, whereas B cells are often aggregated. Inflammatory cells may also be present in Bowman's capsule, resulting in Bowman's capsulitis [24] in acute TCMR.

Notably, interstitial inflammation itself is a non-specific finding. Minimal inflammation may result from acute tubular injury and drug toxicity. In addition, interstitial inflammation can be present in drug-induced interstitial nephritis and polyomavirus-associated interstitial nephritis.

\section{Interstitial hemorrhage}

Interstitial hemorrhage occurs when extravasated red blood cells are present in the interstitium (Fig. 9). This may be seen in, but is not pathognomonic of, active ABMR or acute TCMR.

Interstitial fibrosis

In interstitial fibrosis, collagen fibrils and extracellular 


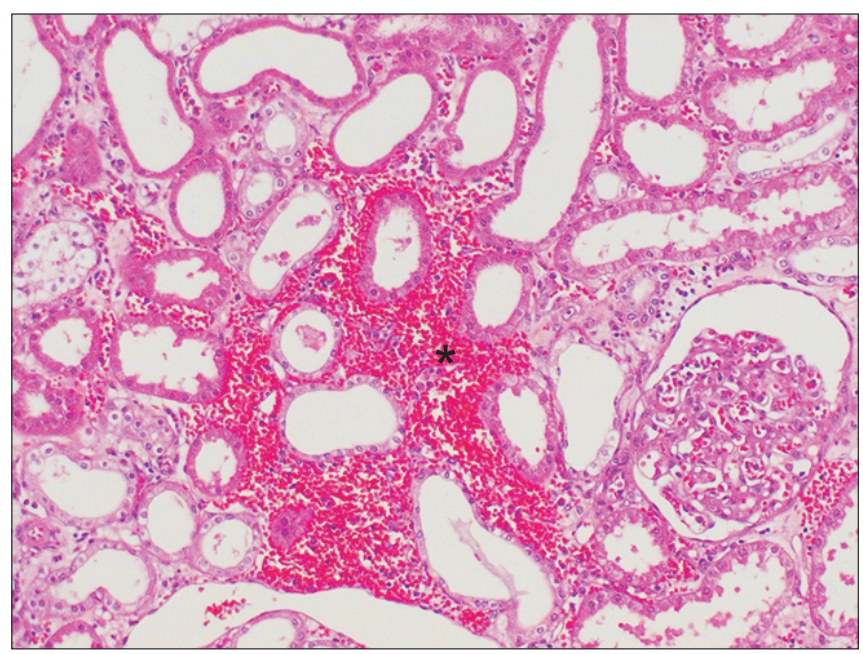

Figure 9. Extravasated red blood cells (asterisk) are found in the interstitium during interstitial hemorrhage; acute tubular injury is also present (H\&E staining, $\times 100)$.

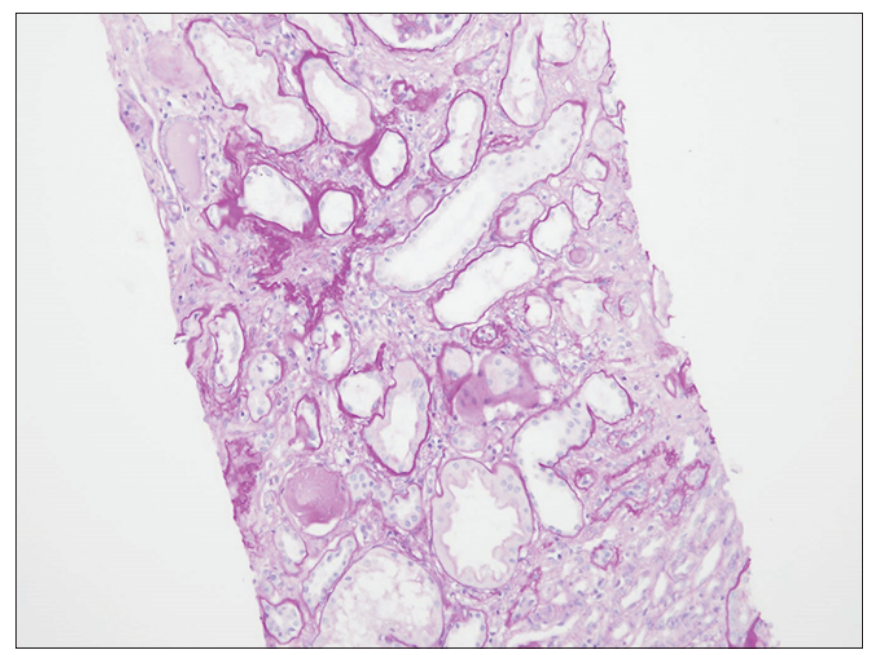

Figure 10. The interstitium is widened by increased extracellular matrix, including collagen fibrils, in interstitial fibrosis (periodic acidSchiff staining, $\times 40)$.

matrix components are increased in the interstitium (Fig. 10). This can be observed in both rejection and non-rejection conditions. In the context of chronic active TCMR, inflammatory cell infiltration may be seen in fibrotic interstitium, together with tubulitis of atrophic and nonatrophic tubules.

\section{Blood vessels}

Peritubular capillaritis

Peritubular capillaritis is characterized by the presence

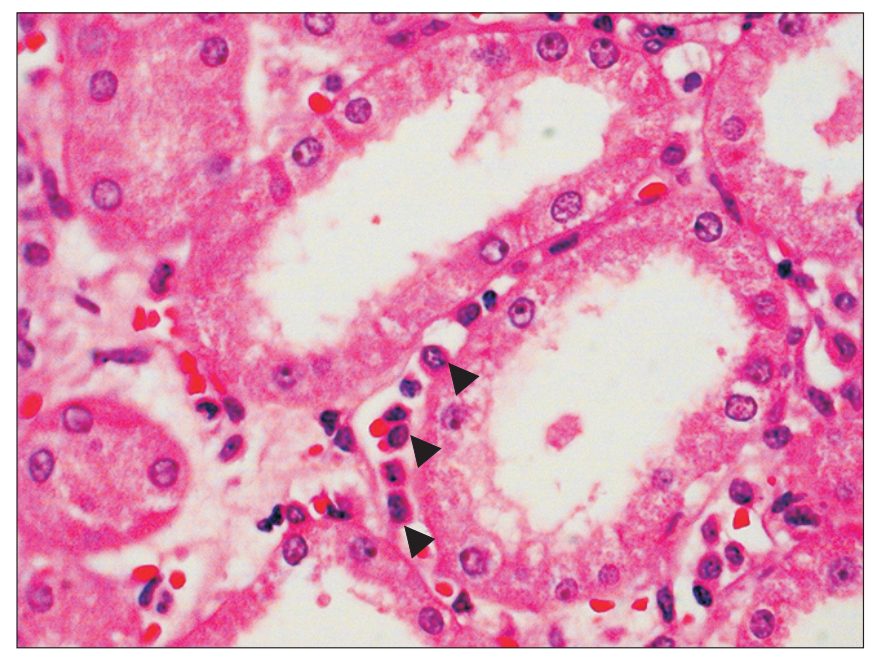

Figure 11. Inflammatory cells (arrowheads) are present in peritubular capillaries (PTCs), and the tubules are relatively intact (H\&E staining, $\times 400)$.

of both mononuclear and polymorphonuclear inflammatory cells in an often-dilated PTC lumen (Fig. 11). This condition is associated with active or chronic active ABMR. Although inflammatory cells may also be found in the PTC lumen in acute TCMR or other conditions, infiltration is usually mild and limited to areas of tubulointerstitial inflammation.

\section{Arteritis}

Arteritis can result from active ABMR and acute TCMR. Intimal arteritis is characterized by the presence of inflammatory cells, mainly $\mathrm{T}$ lymphocytes and macrophages, in the intima, often with lifting off endothelial cells (Fig. 12). In severe cases, transmural inflammation involving arterial media develops, accompanied by fibrinoid necrosis. If the intima does not fully recover to its original state, for example during chronic rejection, the intima will become thickened, forming neointima, which can trap inflammatory cells.

\section{Multilayering of PTC basement membranes (PTCBML)}

PTCBML is associated with chronic ABMR. This condition is usually detected by electron microscopy (Fig. 13), although thickening of capillary basement membranes can be seen by light microscopy in severe cases [25]. Similar to transplant glomerulopathy, PTCBML is believed to be the sequela of repeated PTC injury and repair, resulting in repeated basement membrane formation with progressive narrowing of the lumen. Severe PTCBML with 


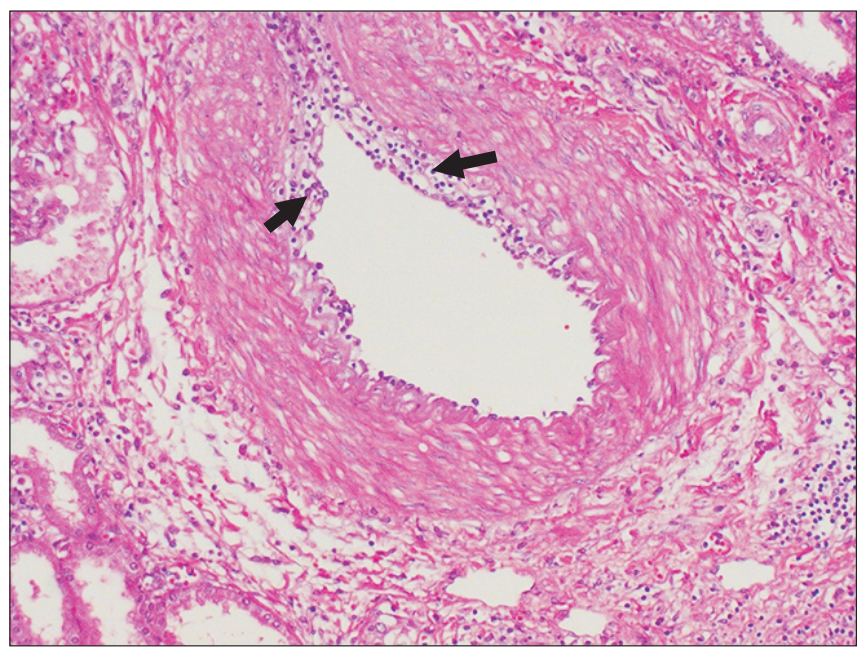

Figure 12. Inflammatory cells (arrows) infiltrate the intima in intimal arteritis, involving more than $50 \%$ of its circumference (H\&E staining, $\times 200$ ).

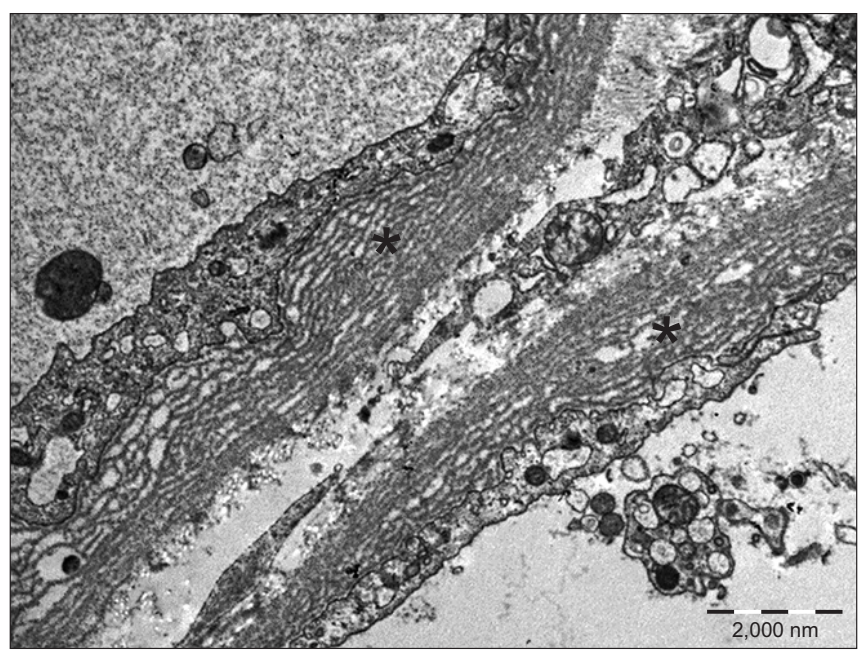

Figure 13. Multilayering of capillary basement membranes (asterisks) is observed in two peritubular capillaries by electron microscopy.

circumferential involvement is more likely to be caused by alloimmune injury, whereas low-grade basement membrane layering with focal involvement is a frequent finding in non-rejections.

\section{Transplant arteriopathy}

Transplant arteriopathy can be caused by chronic ABMR and TCMR. In transplant arteriopathy, the arterial intima is thickened, forming a neointima (Fig. 14), which often contains foamy macrophages and lymphocytes. However, unlike fibroelastosis of hypertensive arterio-

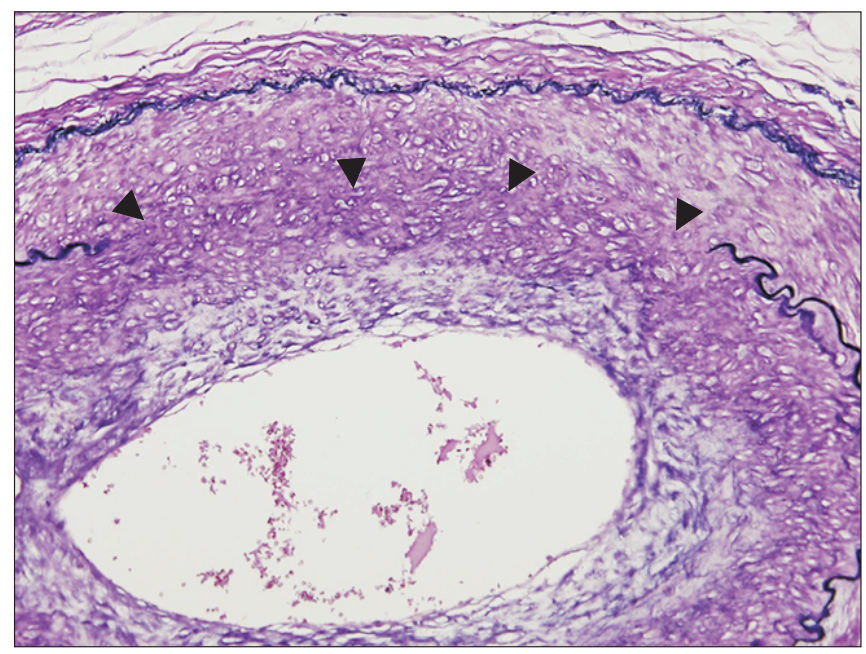

Figure 14. Disruption of the internal elastic lamina (arrowheads) is detected in transplant arteriopathy (elastin staining, $\times 400$ ).

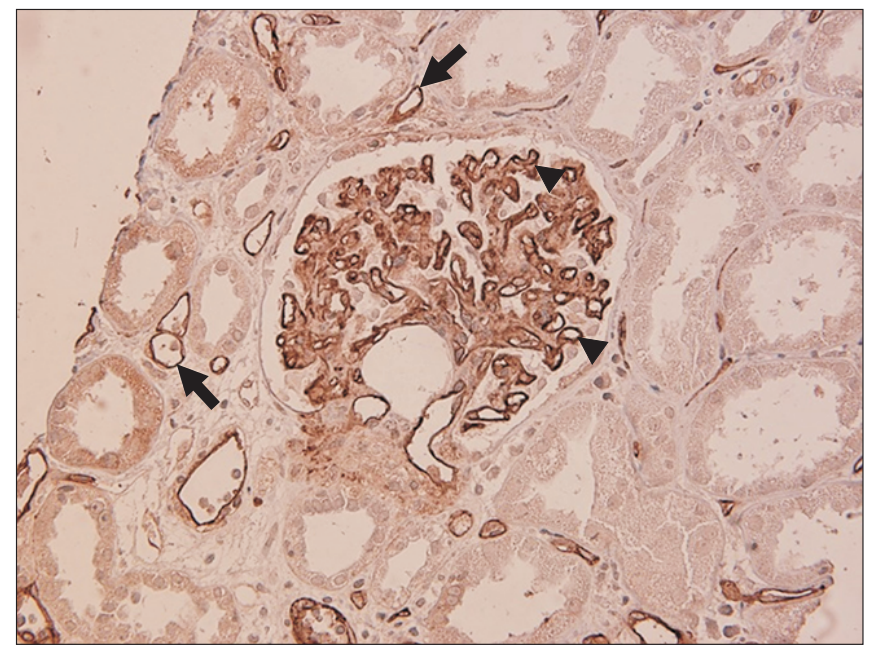

Figure 15. C4d staining is present in peritubular capillaries (arrows) and glomerular capillary walls (arrowheads), as detected by immunohistochemistry $(\times 200)$.

sclerosis, elastic lamellation is not seen in transplant arteriopathy-associated neointima.

\section{PTC C4d staining}

Complement $\mathrm{C} 4 \mathrm{~d}$ is a product of complement activation, and linear, circumferential staining of this molecule in PTCs and the vasa recta (Fig. 15) can be used as a marker for ABMR. C4d binds to the endothelial surface via covalent bonding even after antibody has disappeared; therefore, it serves as a footprint for the antigenantibody reaction. Glomerular C4d staining is more frequently observed in C4d-positive cases than in C4d- 
negative cases, although it has no clinical significance.

Evaluation of C4d positivity varies according to staining method. Immunofluorescence is more sensitive than immunohistochemical methods, and the staining intensity may also be affected by the length of time C4d was present at the antigen-antibody reaction site [26]. ABMR can occur without C4d positivity (C4d-negative ABMR), and C4d staining may be positive without rejection in $\mathrm{ABO}$ incompatible grafts.

\section{Banff classification}

The Banff classification for transplant pathology, which was first published in 1993 [8], has several important advantages: 1) It helps facilitate effective communication among transplant physicians, surgeons, and pathologists; 2 ) it is useful for assessing the effects of new immunosuppressive drugs in international multicenter clinical trials; and 3) it can be used for transplant research in addition to diagnosis.

The most recent Banff classification has six categories (Table 1) [27]. Rejection categories are Category 2 (antibody-mediated changes), Category 3 (borderline for acute TCMR), and Category 4 (TCMR). Before describing the different types of rejection, the basic requirements for biopsy interpretation and histologic scoring systems will be outlined.

\section{Specimen adequacy}

Requirements for specimen adequacy were established in Banff 1991 and Banff 1997. Currently, the presence of seven glomeruli with one artery is marginal, and $\geq 10$ glomeruli with at least two arteries is considered adequate for numeric coding [9]. At least seven slides, three stained with hematoxylin and eosin (H\&E), three stained with PAS, and one stained with trichrome stain should be evaluated.

\section{Quantitative criteria for acute and chronic changes}

Quantitative criteria for classifying acute and chronic changes in glomeruli, tubules, interstitium, and arteries/arterioles were established in 1991 and 1997 [9]. Only cortical tubules and interstitium are considered for evaluation.

Characters representing glomerular changes are ' $\mathrm{g}$ ' (glomerulitis), 'mm' (mesangial matrix increase), and 'cg' (transplant glomerulopathy). Those for tubules are ' $\mathrm{t}$ ' (tubulitis) and 'ct' (tubular atrophy), while for interstitium, they are 'i' (interstitial inflammation) and 'ci' (interstitial fibrosis). The 'ti' and 'i-IFTA (interstitial fibrosis and tubular atrophy)' characters, which indicate tubulointerstitial inflammation, were added in Banff 2007 and Banff 2015, respectively. 'ti' is defined as inflammation in total parenchyma, including scarred and non-scarred cortex [13], whereas 'i-IFTA' is defined as inflammation in areas of tubular atrophy and interstitial fibrosis [17].

For vessels, 'v' (intimal arteritis) and 'cv' (arterial fibrous intimal thickening) describe arteries, and 'ah' and 'aah' indicate hyaline arteriolar thickening [13]. In particular, although 'aah' was added to specifically address calcineurin inhibitor-related arteriolopathy, its specificity has not yet been verified. Two PTC criteria, 'ptc' and 'C4d', were added in Banff 2003 [11] and Banff 2001 [10], respectively, and refined in Banff 2007 [13]. 'Ptc' denotes peritubular capillaritis. ' $\mathrm{C} 4 \mathrm{~d}$ ' is determined by either immunofluorescence or immunohistochemical methods, with different standards for positivity [13].

The Banff scoring system has three grades: mild (1), moderate (2), and severe (3). The cut-off points for ' $\mathrm{g}$ ', 'cg,' 'mm,' 'ct,' 'i', and 'ci' scores are $<25 \%, 25 \%$ to $50 \%$, and $>50 \%$. However, the cut-off points for ' $i$ ' are $10 \%$ to $25 \%, 25 \%$ to $50 \%$, and $>50 \%$, and for 'ci' they are $5 \%$ to $25 \%, 25 \%$ to $50 \%$, and $>50 \%$. Cut-off point for ' $\mathrm{t}$ ' and 'ptc' scores are $<4,4$ to 10 , and $>10$ inflammatory cells/tubule or PTC $[11,13]$. Scores for ' $v$ ' are dependent on the extent of vascular wall involvement ( $<25 \%$ intima, $>25 \%$ intima, and medial involvement), and for 'cv', they are dependent on the percentage of luminal narrowing $(<25 \%$, $25 \%$ to $50 \%$, and $>50 \%$ ). Scores for 'ah' are dependent on the number and severity of hyalinosis. An asterisk $\left({ }^{*}\right)$ is added to express the type of inflammatory cells ('i', 'ptc'), the presence of interstitial hemorrhage or infarction (' $v$ '), or arteriolitis ('ah') [27].

Table 1. Banff diagnostic categories (modified from reference [27])

\begin{tabular}{ll} 
Category 1 & Normal biopsy or non-specific changes \\
Category 2 & Antibody-mediated changes \\
Category 3 & Suspicious (borderline) for acute T cell-mediated rejection \\
Category 4 & T cell-mediated rejection \\
Category 5 & Interstitial fibrosis and tubular atrophy \\
Category 6 & Other non-rejection changes \\
\hline
\end{tabular}


Table 2. Antibody-mediated rejection (modified from reference [27])

\begin{tabular}{|c|c|c|c|}
\hline & Morphologic & Immunologic & Serologic \\
\hline \multirow[t]{4}{*}{ Acute ABMR } & Microvascular inflammation ( $g>0$ and/or ptc $>0$ ) & Linear C4d staining in PTCs & DSAs \\
\hline & Intimal or transmural arteritis $(v>0)$ & At least moderate microvascular inflammation $([g+p t c] \geq 2)$ & \\
\hline & Acute TMA in the absence of any other cause & $\begin{array}{l}\text { Increased expression of gene transcripts in biopsy tissue } \\
\text { indicative of endothelial injury }\end{array}$ & \\
\hline & $\begin{array}{l}\text { Acute tubular injury in the absence of any other } \\
\text { apparent cause }\end{array}$ & & \\
\hline \multirow{3}{*}{$\begin{array}{l}\text { Chronic active } \\
\text { ABMR }\end{array}$} & TG (cg > 0), if no evidence of chronic TMA & Linear C4d staining in PTCs & DSAs \\
\hline & Severe PTC basement membrane multilayering & At least moderate microvascular inflammation $([g+p t c] \geq 2)$ & \\
\hline & $\begin{array}{l}\text { Arterial intimal fibrosis of new onset, excluding } \\
\text { other causes }\end{array}$ & $\begin{array}{l}\text { Increased expression of gene transcripts in the biopsy } \\
\text { tissue indicative of endothelial injury }\end{array}$ & \\
\hline \multirow[t]{3}{*}{ Chronic ABMR } & TG (cg > 0), if no evidence of chronic TMA & $\begin{array}{l}\text { A prior documented diagnosis of active or chronic active } \\
\text { ABMR }\end{array}$ & DSAs \\
\hline & Severe PTC basement membrane multilayering & & \\
\hline & $\begin{array}{l}\text { Arterial intimal fibrosis of new onset, excluding } \\
\text { other causes }\end{array}$ & & \\
\hline
\end{tabular}

ABMR, antibody-mediated rejection; cg, transplant glomerulopathy; DSA, donor-specific antibody; g, glomerulitis; PTC, peritubular capillary; TMA, thrombotic microangiopathy; $v$, intimal arteritis.

\section{Antibody-mediated rejection}

Diagnosis of active and chronic ABMR requires the presence of morphologic, immunohistologic, and serologic evidence. The morphologic features suggestive of active and chronic injury are unique, but active injury may coexist in chronic active ABMR. In contrast, the immunohistologic and serologic indicators are the same for active and chronic ABMR (Table 2) [27].

Although C4d was believed to be a distinct immunohistologic marker for ABMR [10,12], it was later recognized that $\mathrm{C} 4 \mathrm{~d}$ deposition can be present without morphologic evidence of active rejection [13], and C4d may be absent in ABMR $[15,16]$. Furthermore, donor-specific antibody (DSA) is not always detected in ABMR $[28,29]$. Therefore, 'Increased expression of gene transcripts/classifiers strongly associated with ABMR' was included as an alternative to C4d positivity in Banff 2017 [18].

The inclusion of ancillary molecular markers for diagnosis/differential diagnosis of ABMR has been supported by several reports that have described discrepancies between clinical, immunologic, and pathologic findings, as well as by the limitations of biopsy interpretation [30,31]. Sellarés et al [32] reported that microarray assessment of endothelial cells or NK gene expression was helpful in the diagnosis of ABMR. O'Connell et al [33], Modena et al [34], and Halloran et al [35] also showed benefits of gene expression profiling in the diagnosis of ABMR and identification of patients at risk of chronic injury. In addition, Reeve et al [36] demonstrated better prediction of graft survival from molecular scores than from histologic diagnoses.

\section{T cell-mediated rejection}

The diagnosis of acute and chronic TCMR largely depends on morphologic changes in the tubulointerstitial compartment. Diagnostic criteria for acute TCMR have not changed significantly since Banff 1991, and are graded according to active inflammation of non-atrophic tubules and interstitium and vessels. However, the criteria for chronic active TCMR were updated in Banff 2017 (Table 3) [27]. To diagnose chronic active TCMR, tubulointerstitial inflammation with a score $>2$ should be present in areas of atrophic tubules and interstitial fibrosis, as well as in non-scarred tubules and interstitium [18,37-39].

\section{Other revisions and Banff working groups}

The term 'CAN' (chronic allograft nephropathy) was used to describe tubular atrophy and interstitial fibrosis, regardless of the underlying pathogenetic mechanisms, in the initial Banff 1991 report [8]. However, this term is problematic due to the fact that both rejections and non- 
Table 3. T cell-mediated rejection (modified from reference [27])

\begin{tabular}{|c|c|c|}
\hline & Description & Banff scores \\
\hline \multicolumn{3}{|c|}{ Acute TCMR } \\
\hline Type IA & Moderate tubulitis and at least moderate interstitial inflammation & $\mathrm{t} 2 \mathrm{i} 2$ or $\mathrm{t} 2 \mathrm{i} 3$ \\
\hline Type IB & Severe tubulitis and at least moderate interstitial inflammation & $\mathrm{t} 3 \mathrm{i} 2$ or $\mathrm{t} 3 \mathrm{i} 3$ \\
\hline Type IIA & Mild to moderate intimal arteritis & $\mathrm{v} 1$ \\
\hline Type IIB & Severe intimal arteritis (> $25 \%$ of the luminal area) & v2 \\
\hline Type III & 'Transmural' arteritis and/or fibrinoid necrosis & v3 \\
\hline \multicolumn{3}{|c|}{ Chronic active TCMR } \\
\hline Grade IA & $\begin{array}{l}\text { Moderate tubulitis and at least moderate total cortical inflammation and at least moderate } \\
\text { scarred cortical inflammation and other known causes ruled out }\end{array}$ & $\begin{array}{l}\mathrm{t} 2, \mathrm{ti} \geq 2, \text { and } \\
\mathrm{i}-\mathrm{IFTA} \geq 2\end{array}$ \\
\hline Grade IB & $\begin{array}{l}\text { Severe tubulitis and at least moderate total cortical inflammation and at least moderate } \\
\text { scarred cortical inflammation and other known causes ruled out }\end{array}$ & $\begin{array}{l}\mathrm{t} 3, \mathrm{ti} \geq 2, \text { and } \\
\mathrm{i}-\mathrm{IFTA} \geq 2\end{array}$ \\
\hline Grade II & Arterial intimal fibrosis with mononuclear cell inflammation, formation of neointima & cv1, cv2, or cv3 \\
\hline
\end{tabular}

$\mathrm{cv}$, arterial fibrous intimal thickening; i, interstitial inflammation; i-IFTA, tubulointerstitial inflammation (inflammation in areas of interstitial fibrosis and tubular atrophy); t, tubulitis; TCMR, T cell-mediated rejection; ti, tubulointerstitial inflammation (inflammation in total parenchyma, including scarred and non-scarred cortex); v, intimal arteritis.

rejections are included within the same group. It was therefore eliminated in Banff 2005 and replaced by specific diagnosis or the non-specific term 'IFTA' (interstitial fibrosis and tubular atrophy) [12].

Specialized Banff working groups have been convened to address issues relating to problematic areas in biopsy interpretation. The first working groups were organized at the Banff 2009 meeting, and these focused on 'Isolated v-lesion', 'Fibrosis scoring,' 'Polyoma virus nephropathy staging, 'Glomerular lesion scoring, 'Molecular pathology', and 'Quality assurance' [14]. At the Banff 2011 and Banff 2013 meetings, the 'C4d-Banff initiative for quality assurance in transplantation' (BIFQUIT), 'BK-BIFQUIT', 'Implantation biopsies', 'C4d-negative ABMR', 'TCMR', 'Clinical and laboratory assessment of highly sensitized patients', and 'Evaluation of adjunctive diagnostics in renal allograft biopsy interpretation' groups were formed $[15,16]$. Working groups on 'Electron microscopy', 'TMA', 'Recurrent glomerular disease,' 'Composite surrogate end points, 'HIV+/HIV+ renal transplants', and 'Banff rules and dissemination' were newly formed at the Banff 2015 and Banff 2017 meetings $[17,18]$. Several reports have been published from the working groups [40-42], and their efforts have been reflected in Banff updates.

\section{Pitfalls of the current Banff classification}

Although significant progress has been made in our understanding of rejection pathogenesis and in the re- finement of morphologic and laboratory criteria for Banff classification, a number of uncertainties and drawbacks remain. These will be discussed in the following sections.

\section{Criteria for quantitative scoring}

In the Banff classification, glomerulitis is defined as endothelial cell enlargement along with the presence of inflammatory cells and capillary occlusion of $\geq 1$ capillary loops. However, the number of inflammatory cells and minimum threshold for near-occlusion have not been clarified [43]. In addition, endothelial enlargement is subjective, and therefore, a mild degree of endothelial activation may not be recognized.

Presently, borderline changes are 'suspicious' for acute TCMR. The criteria for diagnosis of tubulitis and interstitial inflammation have also been changed at a number of meetings, resulting in confusion and inconsistent communication. For example, at Banff 2001, the criteria were 'tl and at least il'. At Banff 2005 they were changed to 'tl, t2, or t 3 with i0 or il', and at Banff 2007, they were modified again to 't1, t2, or $\mathrm{t} 3$ with $\mathrm{i} 0$ or il' or 'i2 or i3 with t1'. Finally, at Banff 2017, the criteria for tubulitis and interstitial inflammation were established as ' $t>0$ with i0 or i1' or 'i2 or i3 with t1'. In a recent report on borderline changes, these inconsistences have resulted in heterogeneous diagnostic groups [44].

Another parameter that may be considered for adjustment is PTCBML. This was defined as $\geq 7$ PTC basement membrane layers in $\geq 1$ PTC, or 5 to 6 layers in $\geq 3$ PTCs. 
However, the minimum numbers and cut-off values of PTCBML are difficult to determine in daily practice [45]. Furthermore, electron microscopy, which is required for accurate diagnosis, is not available in many transplant centers.

\section{Non-rejection conditions}

Non-rejection conditions/diseases are grouped in Banff category 6 as a separate sheet [8]. However, with increasing graft survival, there have been increased opportunities to observe these conditions in late post-transplant biopsies, and they are often associated with graft dysfunction. For example, development of massive crescents in post-transplant IgA nephropathy or recurrent antineutrophil cytoplasmic antibody-associated glomerulonephritis may be a major contributor to graft dysfunction [46-48]. Additionally, recurrent focal segmental glomerulosclerosis can cause massive proteinuria. Therefore, integrated evaluation and interpretation of dominant injury patterns are required, particularly in cases with combined non-rejection conditions/diseases and rejections. Further, 'g,' 'cg', and ' $m m$ ' scores are used in the classification of both rejection and glomerular diseases $[49,50]$, but scores for crescents and global and segmental sclerosis, which are useful in the evaluation of glomerular diseases, are not included in the Banff parameters.

\section{Uncertain pathogenesis for similar morphologies}

The histology of intimal arteritis and transplant arteriopathy is rather distinct. However, it is difficult to determine whether the development of intimal arteritis and transplant arteriopathy is associated with cell or antibody-mediated mechanisms or if these conditions arose with or without rejection. One morphologic clue is the presence of other known features of TCMR or ABMR; however, this does not apply in cases of isolated vasculitis. Notably, inflammatory cell phenotyping in isolated vasculitis has revealed heterogeneity [51-53], which may reflect distinct injury mechanisms.

The observation of ' $\mathrm{C} 4 \mathrm{~d}$-positive renal transplant biopsies without evidence of rejection' is also associated with uncertainty. Intriguingly, one study performed gene expression analysis and identified patients at higher risk of developing ABMR among these cases [54].

\section{Others}

In the current Banff classification, interstitial inflammation is defined by its extent. However, there is no way to determine whether inflammatory cells are active or quiescent or if their composition is related to inflammatory activity or fibrosis. Further, asterisks in some Banff scores, some related to inflammatory subtypes, are not popular, and their significance is not known.

\section{Future perspectives}

The discovery and application of non-invasive molecular markers for effective diagnosis of renal transplant rejection are active fields of research [55-57]. Importantly, to develop a comprehensive approach, integration of tissue pathology assessment, transcriptomics, and urinary proteomics may be needed, particularly in cases of chronic TCMR [58]. Recently, the NanoString Banff consortium was launched to develop the nCounter Human Organ Transplant Panel to identify biomarkers of rejection, uncover the mechanisms behind tissue damage, and monitor immunosuppressive drug toxicity and infections using paraffin-embedded renal tissue [59]. Diagnosis of mixed rejection and the often problematic coexistence of polyomavirus nephropathy and rejection or calcineurin inhibitor toxicity and rejection may benefit from this approach. Furthermore, clinical significance of antibody responses to non-HLA antigens, such as major histocompatibility complex class I chain-related proteins $\mathrm{A}$ and $\mathrm{B}$ and angiotensin II type 1 receptor, may be clarified $[60,61]$.

Immunophenotyping of allograft biopsies may also provide some additional benefits, for example facilitating the detection of glomerulitis. In particular, this strategy may help us to understand and distinguish the inflammatory and profibrotic phases of inflammation and/or the activity of inflammation. To this end, multiplex immunohistochemistry is likely to be especially useful, as inflammatory cells are mixed in nature and vary throughout the rejection process [62-64]. Digital pathology has become increasingly popular in pathology practice, both in clinical biopsy diagnosis and in research. This strategy has the potential to reduce inter-observer variation and increase objectivity through use of quantification. For example, interstitial inflammatory phenotypes have been reported from digitization of immunostained biopsy sections and 
algorithm-driven analysis, with good clinical correlation [65]. Deep learning-based histopathological assessment of transplant biopsies [66] has also been performed, and high correlations between the neural network's quantification of glomeruli, interstitium, and atrophic tubules and results from renal pathologists were reported.

As noted previously, addition of scores for crescents and global and segmental sclerosis to the current Banff scores is necessary for integrated evaluation of biopsies from patients with combined non-rejection conditions/ diseases and rejections.

Lastly, with recent advances in bioengineered organs, we may also need to implement strategies to deal with abnormalities in bioengineered organs. Recently, Solez et al [67] proposed the construction of a framework for the classification of tissue engineering pathology at future Banff Transplant Pathology meetings, in collaboration with the Human Cell Atlas project [68].

To summarize, for precision diagnosis of transplant rejection, integration of histological, laboratory, and tailored molecular markers is necessary. Artificialintelligence based assistance has the potential to further improve diagnostic accuracy.

\section{Conflicts of interest}

The author has no conflicts of interest to declare.

\section{Acknowledgments}

I would like to express my gratitude to professors $\mathrm{Yu}$ Seun Kim, Soon Il Kim, Myoung Soo Kim, Beom Suk Kim, Kyu Ha Huh, and Beom Jin Lim, as well as to registered nurse Kyung Ock Jeon and the transplant team of Yonsei University Severance Hospital for their efforts and devotion to renal transplantation.

\section{References}

[1] Doyle AM, Lechler RI, Turka LA. Organ transplantation: halfway through the first century. J Am Soc Nephrol 2004; 15:2965-2971.

[2] ESRD Registry Committee, Korean Society of Nephrology. Current renal replacement therapy in Korea - Insan Memorial Dialysis Registry, 2018. Available from: http://www.ksn. or.kr/rang_board/list.html?code=sinchart.
[3] Sellarés J, de Freitas DG, Mengel M, et al. Understanding the causes of kidney transplant failure: the dominant role of antibody-mediated rejection and nonadherence. Am J Transplant 2012;12:388-399.

[4] Lindquist RR, Guttmann RD, Merrill JP, Dammin GJ. Human renal allografts. Interpretation of morphologic and immunohistochemical observations. Am J Pathol 1968;53:851881.

[5] Glassock RJ, Feldman D, Reynolds ES, Dammin GJ, Merrill JP. Human renal isografts: a clinical and pathologic analysis. Medicine (Baltimore) 1968;47:411-454.

[6] Kincaid-Smith P. Histological diagnosis of rejection of renal homografts in man. Lancet 1967;2:849-852.

[7] Millard PR, Herbertson BM, Evans DB. Renal biopsy in clinical management of renal transplantation. A lightmicroscopy study. Lancet 1970;1:113-116.

[8] Solez K, Axelsen RA, Benediktsson H, et al. International standardization of criteria for the histologic diagnosis of renal allograft rejection: the Banff working classification of kidney transplant pathology. Kidney Int 1993;44:411-422.

[9] Racusen LC, Solez K, Colvin RB, et al. The Banff 97 working classification of renal allograft pathology. Kidney Int 1999;55:713-723.

[10] Racusen LC, Colvin RB, Solez K, et al. Antibody-mediated rejection criteria - an addition to the Banff 97 classification of renal allograft rejection. Am J Transplant 2003;3:708-714.

[11] Racusen LC, Halloran PF, Solez K. Banff 2003 meeting report: new diagnostic insights and standards. Am J Transplant 2004;4:1562-1566.

[12] Solez K, Colvin RB, Racusen LC, et al. Banff '05 meeting report: differential diagnosis of chronic allograft injury and elimination of chronic allograft nephropathy ('CAN'). Am J Transplant 2007; 7:518-526.

[13] Solez K, Colvin RB, Racusen LC, et al. Banff 07 classification of renal allograft pathology: updates and future directions. Am J Transplant 2008;8:753-760.

[14] Sis B, Mengel M, Haas M, et al. Banff '09 meeting report: antibody mediated graft deterioration and implementation of Banff working groups. Am J Transplant 2010;10:464-471.

[15] Mengel M, Sis B, Haas M, et al.; Banff meeting report writing committee. Banff 2011 meeting report: new concepts in antibody-mediated rejection. Am J Transplant 2012;12:563570.

[16] Haas M, Sis B, Racusen LC, et al.; Banff meeting report writing committee. Banff 2013 meeting report: inclusion of c4d-negative antibody-mediated rejection and antibody- 
associated arterial lesions. Am J Transplant 2014;14:272283.

[17] Loupy A, Haas M, Solez K, et al. The Banff 2015 kidney meeting report: current challenges in rejection classification and prospects for adopting molecular pathology. Am J Transplant 2017;17:28-41.

[18] Haas M, Loupy A, Lefaucheur C, et al. The Banff 2017 kidney meeting report: revised diagnostic criteria for chronic active $\mathrm{T}$ cell-mediated rejection, antibody-mediated rejection, and prospects for integrative endpoints for nextgeneration clinical trials. Am J Transplant 2018;18:293-307.

[19] Van Loon E, Lerut E, Naesens M. The time dependency of renal allograft histology. Transpl Int 2017;30:1081-1091.

[20] Working Group of the International IgA Nephropathy Network and the Renal Pathology Society, Roberts IS, Cook HT, et al. The Oxford classification of IgA nephropathy: pathology definitions, correlations, and reproducibility. Kidney Int 2009;76:546-556.

[21] Remuzzi G, Bertani T. Renal vascular and thrombotic effects of cyclosporine. Am J Kidney Dis 1989;13:261-272.

[22] Laszik ZG, Kambham N, Silva FG. Thrombotic microangiopathies. In: Jennette JC, Olson JL, Silva FG, D'Agati VD, eds. Heptinstall's pathology of the kidney. 7th ed. Philadelphia: Lippincott Williams \& Wilkins, 2014.

[23] Sartelet H, Toupance O, Lorenzato M, et al. Sirolimusinduced thrombotic microangiopathy is associated with decreased expression of vascular endothelial growth factor in kidneys. Am J Transplant 2005;5:2441-2447.

[24] Gallan AJ, Chon WJ, Josephson MA, Cunningham PN, Henriksen KJ, Chang A. Bowman capsulitis predicts poor kidney allograft outcome in T cell-mediated rejection. Hum Pathol 2018;76:47-51.

[25] Aita K, Yamaguchi Y, Horita S, et al. Thickening of the peritubular capillary basement membrane is a useful diagnostic marker of chronic rejection in renal allografts. Am J Transplant 2007;7:923-929.

[26] Nickeleit V, Zeiler M, Gudat F, Thiel G, Mihatsch MJ. Detection of the complement degradation product $\mathrm{C} 4 \mathrm{~d}$ in renal allografts: diagnostic and therapeutic implications. J Am Soc Nephrol 2002;13:242-251.

[27] Roufosse C, Simmonds N, Clahsen-van Groningen M, et al. A 2018 reference guide to the Banff classification of renal allograft pathology. Transplantation 2018;102:1795-1814.

[28] Lesage J, Noël R, Lapointe I, et al. Donor-specific antibodies, C4d and their relationship with the prognosis of transplant glomerulopathy. Transplantation 2015;99:69-76.
[29] Senev A, Coemans M, Lerut E, et al. Histological picture of antibody-mediated rejection without donor-specific antiHLA antibodies: clinical presentation and implications for outcome. Am J Transplant 2019;19:763-780.

[30] Halloran PF, Reeve JP, Pereira AB, Hidalgo LG, Famulski KS. Antibody-mediated rejection, T cell-mediated rejection, and the injury-repair response: new insights from the genome Canada studies of kidney transplant biopsies. Kidney Int 2014;85:258-264.

[31] Schinstock CA, Sapir-Pichhadze R, Naesens M, et al. Banff survey on antibody-mediated rejection clinical practices in kidney transplantation: Diagnostic misinterpretation has potential therapeutic implications. Am J Transplant 2019; 19:123-131.

[32] Sellarés J, Reeve J, Loupy A, et al. Molecular diagnosis of antibody-mediated rejection in human kidney transplants. Am J Transplant 2013;13:971-983.

[33] O'Connell PJ, Zhang W, Menon MC, et al. Biopsy transcriptome expression profiling to identify kidney transplants at risk of chronic injury: a multicentre, prospective study. Lancet 2016;388:983-993.

[34] Modena BD, Kurian SM, Gaber LW, et al. Gene expression in biopsies of acute rejection and interstitial fibrosis/tubular atrophy reveals highly shared mechanisms that correlate with worse long-term outcomes. Am J Transplant 2016; 16:1982-1998.

[35] Halloran PF, Reeve J, Akalin E, et al. Real time central assessment of kidney transplant indication biopsies by microarrays: the INTERCOMEX study. Am J Transplant 2017; 17:2851-2862.

[36] Reeve J, Böhmig GA, Eskandary F, et al.; MMDx-Kidney study group. Assessing rejection-related disease in kidney transplant biopsies based on archetypal analysis of molecular phenotypes. JCI Insight 2017;2:94197.

[37] Lefaucheur C, Gosset C, Rabant M, et al. T cell-mediated rejection is a major determinant of inflammation in scarred areas in kidney allografts. Am J Transplant 2018;18:377-390.

[38] Lefaucheur C, Viglietti D, Loupy A. Recognition of i-IF/TA as a component of the $\mathrm{T}$ cell-mediated rejection spectrum: unselected population approach vs random case selection. Am J Transplant 2018;18:771-772.

[39] Nankivell BJ, Shingde M, Keung KL, et al. The causes, significance and consequences of inflammatory fibrosis in kidney transplantation: the Banff i-IFTA lesion. Am J Transplant 2018;18:364-376.

[40] Sis B, Bagnasco SM, Cornell LD, et al.; Banff Working 
Group. Isolated endarteritis and kidney transplant survival: a multicenter collaborative study. J Am Soc Nephrol 2015; 26:1216-1227.

[41] Nickeleit V, Singh HK, Randhawa P, et al.; Banff Working Group on Polyomavirus Nephropathy. The Banff Working Group classification of definitive polyomavirus nephropathy: morphologic definitions and clinical correlations. J Am Soc Nephrol 2018;29:680-693.

[42] Farris AB, Chan S, Climenhaga J, et al. Banff fibrosis study: multicenter visual assessment and computerized analysis of interstitial fibrosis in kidney biopsies. Am J Transplant 2014;14:897-907.

[43] Ozluk Y, Caliskan Y, Sevinc M, et al. Re-evaluation of glomerulitis using occlusion criteria based on the Banff 2013 revision: a retrospective study. Transpl Int 2017;30:579-588.

[44] Nankivell BJ, Agrawal N, Sharma A, et al. The clinical and pathological significance of borderline $\mathrm{T}$ cell-mediated rejection. Am J Transplant 2019;19:1452-1463.

[45] Go H, Shin S, Kim YH, Han DJ, Cho YM. Refinement of the criteria for ultrastructural peritubular capillary basement membrane multilayering in the diagnosis of chronic active/ acute antibody-mediated rejection. Transpl Int 2017;30: 398-409.

[46] Jeong HJ, Kim YS, Kwon KH, et al. Glomerular crescents are responsible for chronic graft dysfunction in post-transplant IgA nephropathy. Pathol Int 2004;54:837-842.

[47] Jeong HJ, Park SK, Cho YM, et al. Progression of renal allograft histology after renal transplantation in recurrent and nonrecurrent immunoglobulin A nephropathy. Hum Pathol 2008;39:1511-1518.

[48] Lim BJ, Joo DJ, Kim MS, et al. Usefulness of Oxford classification in assessing immunoglobulin A nephropathy after transplantation. Transplantation 2013;95:1491-1497.

[49] Schrezenmeier E, Wu K, Halleck F, et al. Successful recovery of acute renal transplant failure in recurrent hepatitis $\mathrm{C}$ virus-associated membranoproliferative glomerulonephritis. Am J Transplant 2017;17:819-823.

[50] Yamakawa T, Kobayashi A, Yamamoto I, et al. Impact of primary diabetic nephropathy on arteriolar hyalinosis lesions in patients following kidney transplantation. Nephrology (Carlton) 2018;23 Suppl 2:70-75.

[51] Lim BJ, Kwon HJ, Bae YS, Jeong HJ. Immunohistochemical analysis of infiltrating inflammatory cells in the isolated vlesion of allograft kidney. Transplant Proc 2015;47:622-625.

[52] Rabant M, Boullenger F, Gnemmi V, et al. Isolated v-lesion in kidney transplant recipients: characteristics, associa- tion with DSA, and histological follow-up. Am J Transplant 2018;18:972-981.

[53] Wohlfahrtova M, Hruba P, Klema J, et al. Early isolated Vlesion may not truly represent rejection of the kidney allograft. Clin Sci (Lond) 2018;132:2269-2284.

[54] Dominy KM, Willicombe M, Al Johani T, et al. Molecular assessment of C4d-positive renal transplant biopsies without evidence of rejection. Kidney Int Rep 2018;4:148-158.

[55] Salcido-Ochoa F, Allen JC Jr. Biomarkers and a tailored approach for immune monitoring in kidney transplantation. World J Transplant 2017;7:276-284.

[56] Bloom RD, Bromberg JS, Poggio ED, et al.; Circulating Donor-Derived Cell-Free DNA in Blood for Diagnosing Active Rejection in Kidney Transplant Recipients (DART) Study Investigators. Cell-free DNA and active rejection in kidney allografts. J Am Soc Nephrol 2017;28:2221-2232.

[57] Madill-Thomsen K, Perkowska-Ptasińska A, Böhmig GA, et al.; MMDx-Kidney Study Group. Discrepancy analysis comparing molecular and histology diagnoses in kidney transplant biopsies. Am J Transplant 2019 Dec 17 [Epub]. DOI: $10.1111 /$ ajt.15752.

[58] Marx D, Metzger J, Olagne J, et al. Proteomics in kidney allograft transplantation-application of molecular pathway analysis for kidney allograft disease phenotypic biomarker selection. Proteomics Clin Appl 2019;13:e1800091.

[59] NanoString. nCounter ${ }^{\circledR}$ Human Organ Transplant Panel [Internet]. Seattle (USA): NanoString. Available from: https://www.nanostring.com/products/gene-expressionpanels/gene-expression-panels-overview.

[60] Filippone EJ, Farber JL. Humoral immune response and allograft function in kidney transplantation. Am J Kidney Dis 2015;66:337-347.

[61] Lefaucheur C, Viglietti D, Bouatou Y, et al. Non-HLA agonistic anti-angiotensin II type 1 receptor antibodies induce a distinctive phenotype of antibody-mediated rejection in kidney transplant recipients. Kidney Int 2019;96:189-201.

[62] Bräsen JH, Khalifa A, Schmitz J, et al. Macrophage density in early surveillance biopsies predicts future renal transplant function. Kidney Int 2017;92:479-489.

[63] Salcido-Ochoa F, Hue SS, Peng S, et al. Histopathological analysis of infiltrating $\mathrm{T}$ cell subsets in acute $\mathrm{T}$ cell-mediated rejection in the kidney transplant. World J Transplant 2017;7:222-234.

[64] Kim J, Choi SE, Lim BJ, et al. Clinical significance of macrophage polarization in antibody-mediated rejection of renal allograft. Transplant Proc 2018;50:1005-1008. 
[65] Sicard A, Meas-Yedid V, Rabeyrin M, et al. Computer-assisted topological analysis of renal allograft inflammation adds to risk evaluation at diagnosis of humoral rejection. Kidney Int 2017;92:214-226.

[66] Hermsen M, de Bel T, den Boer M, et al. Deep learningbased histopathologic assessment of kidney tissue. J Am Soc Nephrol 2019;30:1968-1979.
[67] Solez K, Fung KC, Saliba KA, et al. The bridge between transplantation and regenerative medicine: Beginning a new Banff classification of tissue engineering pathology. Am J Transplant 2018;18:321-327.

[68] Moghe I, Loupy A, Solez K. The human cell atlas project by the numbers: relationship to the Banff classification. Am J Transplant 2018;18:1830. 